\title{
La situation agricole et alimentaire mondiale : causes, conséquences, perspectives
}

\author{
Marcel MAZOYER \\ AgroParisTech, Université Paris XI, \\ Chaire Francqui internationale, \\ Universités francophones belges \\ $<$ marmaz2@tele2.fr>
}

Quelle est précisément l'étendue des insuffisances agricoles et alimentaires présentes? Quelles en sont les causes ? Les conséquences? Les perspectives? Quelles propositions de politiques peut-on faire pour y remédier au plus vite et durablement?

Telles sont les questions auxquelles nous essayons de répondre ici.

\section{Quelle est I'étendue des insuffisances agricoles et alimentaires présentes ?}

\section{La production agricole s'accroît un peu plus vite que la population}

De 1 milliard d'êtres humains en 1800, la population mondiale est passée à 2 milliards en 1925, à 4 milliards en 1975, à 6 milliards en 2000, et elle approcherait les 9 milliards en 2050, pour plafonner autour de 9,5 milliards en 2070-80. L'explosion démographique, qui s'est propagée dans le monde depuis deux siècles, se ralentit de proche en proche et elle s'arrêtera probablement avant la fin du XXI ${ }^{\mathrm{e}}$ siècle. Au cours de la seconde moitié du XXe siècle, la population mondiale a été multipliée par 2,4 tandis que, dans le même temps, la production agricole et alimentaire mondiale a été multipliée par 2,6. Au plus fort de l'explosion démographique, contrairement aux prévisions de Malthus, la production agricole a donc augmenté plus vite que la population [1].

Pour $77 \%$, cette croissance de la production est due à l'augmentation des rendements : le rendement moyen mondial des cultures a été multiplié par plus de deux en un demi-siècle. Du fait de l'utilisation des semences sélectionnées à haut rendement potentiel, des engrais minéraux et des pesticides, ainsi qu'à l'irriga-

\begin{abstract}
This article presents an overview of the development of agri-food system in the world, the disparity in productivity and income the long terme downward trend in international agricultural prices, the world agricultural divide and its conséquences in terms of poverty and malnutrition. Given this situation of poor farmers it analyses what should be the components of an effective policy development.
\end{abstract}

Key words: agricultural and food situation worldwide, productivity, agricultural prices, poverty, malnutrition

tion qui a été étendue de 80 millions d'hectares en 1950 à 240 millions en 2 000, le rendement moyen a beaucoup plus que doublé dans la plupart des régions des pays industrialisés, dans beaucoup de régions des pays émergents et dans quelques régions des autres pays en développement. Dans de rares régions il a même décuplé, pour atteindre 10 tonnes d'équivalent-céréales ${ }^{1}$ par hectare et se rapprocher ainsi du maximum possible. Par contre, dans d'autres régions des pays en développement et en particulier dans la plupart des régions des pays les moins avancés (expression désignant les 50 pays les plus pauvres du monde), le rendement moyen n'a que peu ou pas augmenté.

Pour $13 \%$, cette augmentation de la production provient de l'extension des terres arables. Ce qui est fort peu.

Et pour les $10 \%$ restant, elle provient de la réduction des périodes de jachère ou de friche entre les cultures, de l'accroissement du nombre de récoltes par an, ainsi que du développement impressionnant, dans quelques régions très peuplées du monde, d'écosystèmes cultivés superposant un ou plusieurs étages d'arboriculture, dominant des associations, denses, de cultures vivrières et fourragères, ainsi que des élevages d'herbivores, de porcs ou de volailles, et parfois même des bassins de pisciculture : des écosystèmes complexes, construits par les paysans, capables de produire, sans engrais minéraux ni pesticides, autant de produits végétaux et animaux que les cultures et les élevages conventionnels pourraient en produire sur la même surface.

\footnotetext{
${ }^{1}$ L'équivalent-céréales est la quantité de céréales ayant la même valeur calorique que la production agricole considérée.
}

Mais, si en un demi-siècle, la croissance de la production agricole a bien été supérieure d'environ $8 \%(2,6: 2,4=1,08)$ à celle de la population, force est de reconnaître qu'elle fut néanmoins insuffisante et trop inégale pour subvenir convenablement aux besoins de toute I'humanité.

\section{Pauvreté et malnutrition}

Sur les 6,6 milliards d'habitants de la planète, on en compte en effet plus de trois milliards qui disposent de moins de 3 dollars par jour et qui se privent plus ou moins de nourriture. Parmi ceux-ci, il s'en trouve plus de deux milliards qui se privent assez pour souffrir de graves malnutritions c'est-à-dire de maladies invalidantes (anémie, déficience mentale, cécité) dues à des carences alimentaires en micronutriments (fer, iode... vitamines A, E, C...). Nombre d'entre eux souffrent aussi de carence en protéines, trop chères pour eux, et parfois d'obésité, due aux sucres et aux graisses, bon marché, qu'ils consomment en excès pour arriver à satiété.

\section{Pauvreté extrême et sous-alimentation chronique}

On compte enfin plus de 1 milliard de personnes qui disposent de moins de 1 dollar par jour, parmi lesquelles il s'en trouve 862 millions (moyenne 2002-2004, dernier chiffre connu) [2] qui souffrent de sous-alimentation chronique, autrement dit qui ont faim une partie de l'année, car faute d'une alimentation suffisante en macronutriments (glucides, lipides, protides), ils ne disposent pas tous les jours des 2150 à 2400 kilocalories alimentaires nécessaires pour maintenir leur poids et pour s'activer normalement. 
Sur ces 862 millions de personnes souffrant de la faim, on en trouve plus de 500 millions en Asie et Pacifique et de 200 millions en Afrique subsaharienne, environ 50 millions en Amérique latine-et-Caraïbes et en Afrique du Nord-et-Proche-Orient; près de 30 millions dans les pays en transition et de 10 millions dans les pays industrialisés.

De plus, on le sait, depuis que les chefs d'État et de gouvernement, réunis à Rome à l'occasion du Sommet mondial de l'alimentation de 1996, se sont donné comme objectif de réduire de moitié le nombre de personnes souffrant de la faim, pour 2015 au plus tard, ce nombre n'a pas diminué. Bien au contraire. En passant de 827 millions en 1996 à 862 millions en 2003 (moyenne 2002-2004), il a augmenté de 5 millions par an, en moyenne. À quoi il faut ajouter que, chaque année, 9 millions de ces pauvres sous-alimentés (dont les deux tiers sont des enfants) meurent effectivement de faim chaque année.

\section{La famine et la mort}

Dans les régions écartées, où prévalent déjà durement la pauvreté et la faim, le moindre accident climatique, biologique, économique ou politique peut en effet provoquer une raréfaction et une augmentation des prix des denrées vivrières assez importante pour que, les plus pauvres ne pouvant plus se procurer de nourriture et les plus sous-alimentés ne pouvant pas supporter de privations supplémentaires, les plus vulnérables d'entre eux en meurent. Et si du fait des conflits, fréquents en ce genre d'occasion, les secours n'arrivent pas, la faim s'aiguise encore et tourne à la famine, qui tue de plus en plus de gens.

Conclusion : trois milliards de pauvres se privent plus ou moins de nourriture; deux milliards souffrent de malnutrition, 862 millions souffrent de la faim, 9 millions en meurent chaque année et, malgré cette surmortalité, le nombre de pauvres sous-alimentés a recommencé d'augmenter.

\section{Pauvreté et exode rural}

Selon la FAO toujours, les trois quarts de ces pauvres sous-alimentés sont des ruraux, parmi lesquels on trouve une très grande majorité de paysans mal outillés, mal situés et mal lotis, et $d^{\prime}$ ouvriers agricoles très peu payés. Quant aux autres, beaucoup d'entre eux sont d'ex-ruraux récemment poussés à l'exode par la pauvreté et par la faim vers les camps de réfugiés et les bidonvilles sous-équipés et sous-industrialisés, où règnent le chômage, les bas salaires et bien d'autres misères matérielles et morales. Enfin, il faut souligner que, malgré un exode rural de plus de 50 millions de personnes par an, le nombre des pauvres sous-alimentés des campagnes ne diminue pas, ce qui signifie qu'un nombre au moins égal de nouveaux pauvres sous-alimentés se forme chaque année dans les campagnes du monde. Certes, il paraît surprenant qu'après plusieurs décennies de révolution agricole contemporaine et de révolution verte, la pauvreté et la sous-alimentation rurale soient toujours aussi massivement répandues. Mais c'est oublier le caractère incroyablement inégal de cet extraordinaire développement.

\section{La fracture agricole mondiale}

Malgré cet exode rural massif, la population rurale, qui s'élève à 3,34 milliards de personnes (soit $53 \%$ de la population mondiale) n'a jamais été si nombreuse. Quant à la population agricole totale (population agricole active et non active) qui s'élève à 2,7 milliards de personnes, elle représente $41 \%$ de la population mondiale. Alors que la population agricole active, qui s'élève à 1,34 milliard de personnes, représente $43 \%$ de la population active du monde [1].

Or, pour ces 1,34 milliard d'actifs agricoles, on ne compte dans le monde que 28 millions de tracteurs et 250 millions d'animaux de travail (soit respectivement $2,1 \%$ et $18,6 \%$ du nombre total des actifs agricoles). Cela signifie que plus de 1 milliard d'actifs agricoles travaillent uniquement avec des outils manuels (houe, bêche, bâton fouisseur, faucille...). Un milliard d'actifs dont la moitié environ soit 500 millions utilisent couramment des semences sélectionnées, des engrais minéraux et des pesticides et tandis que l'autre moitié, soit 500 millions d'actifs (ce qui correspond à 1 milliard de bouches à nourrir) n'en utilise pratiquement pas.

Encore faut-il ajouter que dans de nombreux pays ex-coloniaux ou ex-communistes $n$ 'ayant pas connu de réforme agraire récente, la majorité de ces paysans sous-équipés sont encore privés de terre par les grands domaines de plusieurs milliers ou dizaines de milliers $d$ 'hectares, de sorte qu'ils ne disposent que d'une superficie très inférieure à celle qu'ils pourraient cultiver et qui leur serait nécessaire pour nourrir leur famille. Ces paysans sont donc obligés d'aller chercher du travail au jour le jour dans les grands domaines, contre des salaires de 1/4 à 3 dollars la journée selon les pays, les régions et les saisons.

Conclusion: dans ces conditions, on comprend pourquoi, dans presque tous les pays du monde, le revenu moyen des paysans est non seulement très inférieur à celui des citadins, mais encore nettement inférieur au salaire de la main-d'œuvre non qualifiée.

\section{Comment a-t-on pu en arriver là ?}

\section{Début du XXe siècle : un écart de productivité de 1 à 10}

Au début du $X X^{e}$ siècle, la plupart des paysans du monde utilisaient exclusivement des outils manuels, et leur productivité du travail ne dépassait pas 1 tonne de céréales ou d'équivalent-céréales par travailleur et par an (1 hectare/travailleur $\times 1$ tonne/hectare). En Europe, dans les colonies de peuplement européennes des régions tempérées et dans certains deltas d'Asie, beaucoup utilisaient cependant des animaux de travail et des matériels tractés, de fabrication artisanale (araire ou charrue, herse, rouleau, charrette, chariot...). Aux États-Unis et en Europe notamment quelques-uns d'entre eux utilisaient déjà les nouvelles machines à traction animale, récemment produites par l'industrie (charruesbrabants, semoirs, faucheuses, faneuses, moissonneuses-lieuses...), grâce auxquelles ils arrivaient à produire jusqu'à 10 tonnes de grain par travailleur et par an (10 ha/tr $\times 1 \mathrm{t} / \mathrm{ha}) . \grave{A}$ l'époque donc, toutes les agricultures du monde s'inscrivaient dans un écart de productivité de l'ordre de 1 à 10.

\section{La révolution agricole contemporaine}

Depuis lors, principalement au cours de la seconde moitié du XX $X^{\mathrm{e}}$ siècle, la révolution agricole contemporaine s'est étendue dans les pays développés et dans quelques secteurs limités des pays en développement [3].

Dans les pays développés, un nombre toujours plus réduit d'exploitations familiales a réussi à franchir toutes les étapes de cette révolution agricole. En céréaliculture par exemple, la puissance des tracteurs et la superficie maximum cultivable par un travailleur ont presque doublé tous les dix ans, pour dépasser aujourd'hui les 200 hectares par travailleur. Dans le même temps, grâce aux semences sélectionnées, aux engrais et aux pesticides, les rendements ont augmenté de plus de 1 tonne par hectare tous les dix ans, pour approcher aujourd'hui les 10 tonnes par hectare dans certaines régions. C'est ainsi que les agriculteurs les mieux équipés et les plus productifs du monde peuvent aujourd'hui produire jusqu'à 2000 tonnes d'équivalent céréales par travailleur et par an (200 ha/tr $\times 10 \mathrm{t} / \mathrm{ha}$ ) (figure 1).

\section{La révolution verte}

D'un autre côté, à partir des années 1960, les agriculteurs des pays en développement qui en avaient les moyens, et qui furent soutenus par des politiques publiques favorables, se sont lan- 


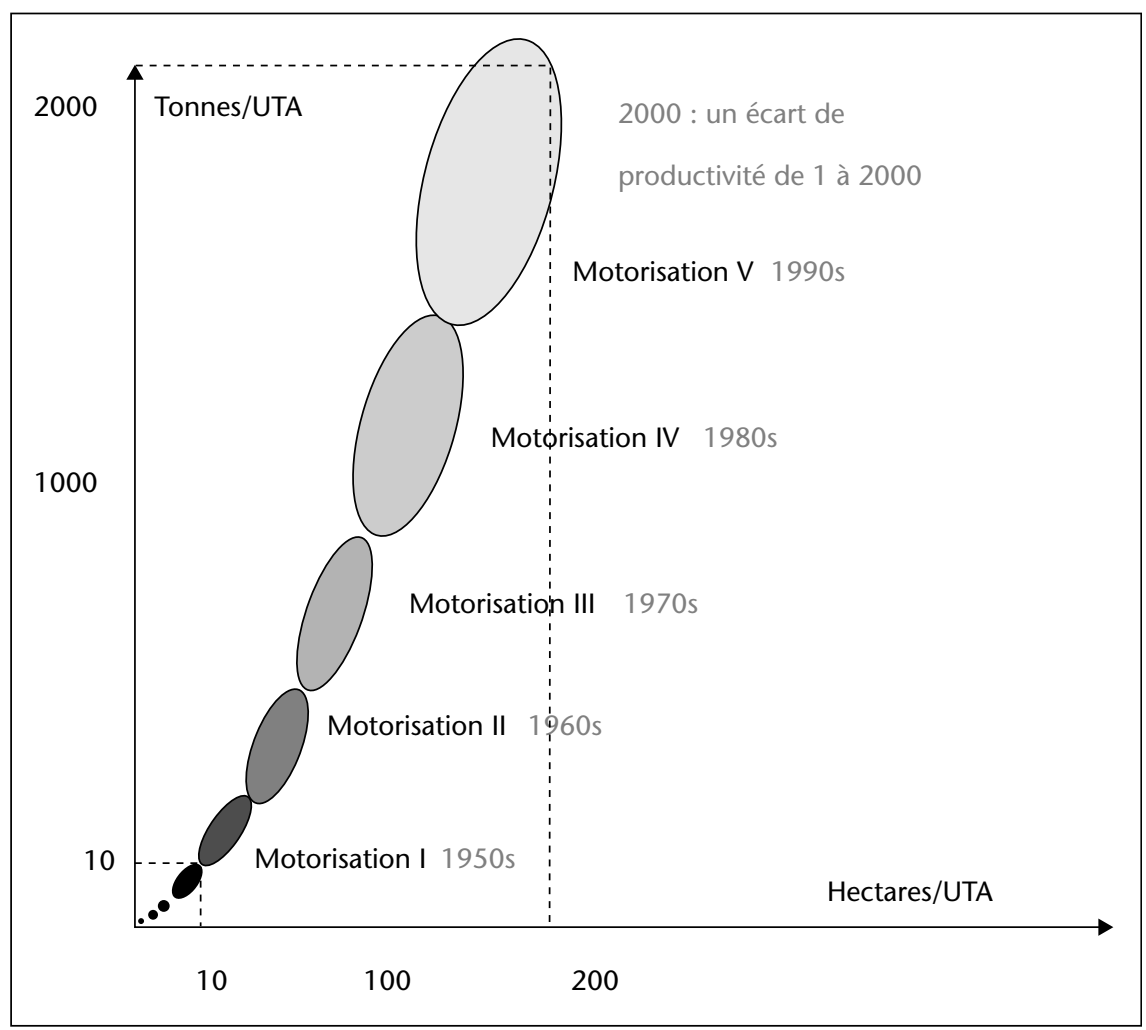

Figure 1. L'accroissement des inégalités de productivité du travail en culture céréalière au xx siècle. Source: $M$. Mazoyer, AgroParisTech.

cés dans la révolution verte [4], une variante de la révolution agricole contemporaine généralement dépourvue de grande motorisationmécanisation. C'est ainsi que dans beaucoup de pays d'Asie, le rendement du riz, qui dépassait rarement 2 tonnes par hectare il y a 40 ans, peut atteindre aujourd'hui 10 tonnes par hectare en une seule récolte, et que la production par hectare et par an peut même approcher les 20 ou 30 tonnes quand les aménagements hydrauliques permettent de faire deux ou trois récoltes dans l'année. En conséquence, un riziculteur cultivant à la main un demihectare et qui produisait 1 tonne de paddy (riz non décortiqué) en 1960 peut en produire selon le cas 5, 10 ou même 15 tonnes aujourd'hui.

\section{Le transfert de la révolution agricole dans les pays de grands domaines et à bas salaires}

De plus, à partir du milieu des années 1970, des investisseurs de toutes sortes (entrepreneurs, grands propriétaires, agro-industrie, agrodistribution, fonds d'investissement...) ont tiré parti de l'expérience de la révolution agricole et de la révolution verte et des hauts prix agricoles du moment (figure 2) pour se lancer dans la modernisation d'anciens grands domaines
2000 tonnes par travailleur, est aujourd'hui de 1 à 2000 .

\section{La baisse tendancielle des prix agricoles internationaux}

\section{Des excédents croissants à prix décroissants}

Dans les pays où la révolution agricole contemporaine et la révolution verte ont le plus progressé, les gains de productivité agricole ont ainsi largement dépassé ceux des autres secteurs de l'économie et, en conséquence, les prix agricoles réels (hors inflation) ont très fortement baissé [3, 5].

Dans les pays développés où la révolution agricole a le plus progressé après 1945, les prix réels des matières premières agricoles et alimentaires de base (céréales, oléoprotéagineux, viandes, lait, œufs...) ont été divisés par 4 ou 5 en un demi-siècle. Dans le même temps, la production végétale ayant augmenté beaucoup plus vite que la population, des quantités croissantes de produits végétaux ont été utilisées par les élevages (volailles, porcins, bovins) dont les produits ont baissé en coût et en prix. Ainsi, malgré une consommation croissante en produits animaux, certains de ces pays bien dotés en terre (États-Unis, Canada, Australie, Nouvelle-Zélande et, dans une moindre mesure, certains pays d'Europe) ont dégagé des excédents exportables en quantités croissantes et à des prix décroissants.

Dans les pays en développement où la révolution verte a le plus progressé, en Asie du Sud, du Sud-Est et de l'Est notamment, même sans grande motorisation, l'augmentation des rendements a entraîné une forte hausse de productivité et une baisse importante des coûts de production et des prix agricoles réels. Et certains de ces pays sont aussi devenus agroexportateurs (Thaillande, Vietnam), alors même que la sous-alimentation y était très répandue.

Dans les anciens pays coloniaux et communistes où les grandes entreprises agricoles à salariés, récemment modernisées, atteignent aujourd'hui un niveau de productivité aussi élevé que celui des exploitations familiales les mieux équipées des pays développés, les coûts de production sont encore plus bas. Dans ces pays en effet, les salaires ne dépassent pas quelques dizaines de dollars par mois, les machines, les engrais, les pesticides fabriqués sur place sont beaucoup moins chers, les charges fiscales sont très faibles et les monnaies locales sont souvent sous-évaluées. Et comme la pauvreté limite les débouchés intérieurs de ces pays, ils peuvent approvisionner les marchés internationaux à 


\section{Dollars US de 1998 le boisseau}

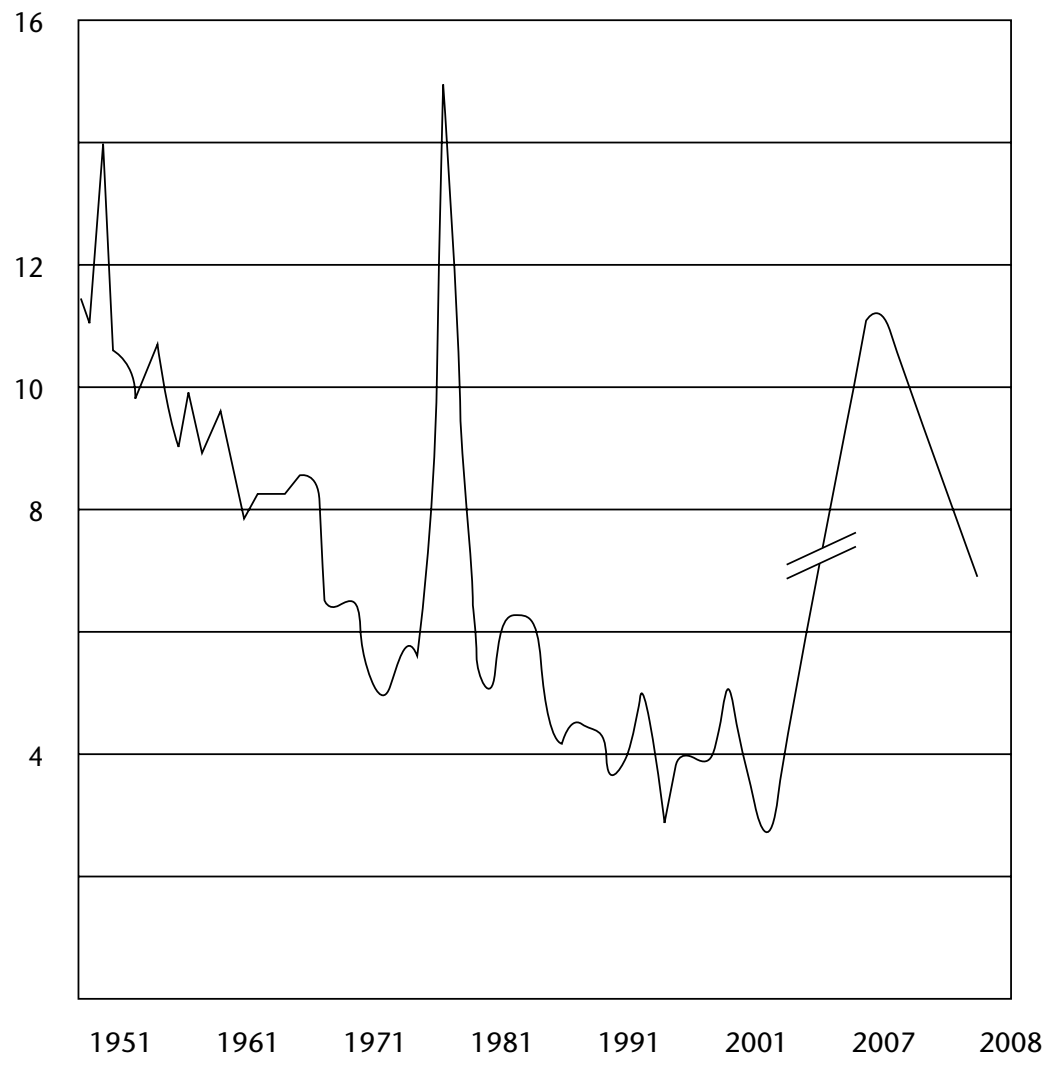

Figure 2. Baisse tendancielle et explosions périodiques du prix international réel du blé (US Dollars constants). Source: Momagri, novembre 2008.

des prix défiant toute concurrence. Ces prix sont d'ailleurs si bas et si rapidement décroissants que même les paysans moyens de ces pays, qui avaient commencé de se moderniser dans les années 1970-80, sont aujourd'hui bloqués dans leur développement, et parfois appauvris au point de rejoindre dans l'exode les millions de petits paysans et d'ouvriers agricoles privés de terre et d'emploi par les grands domaines, qui continuent de s'équiper et de gagner du terrain et des parts de marché (figure 2).

\section{La très forte baisse à long terme des prix agricoles internationaux}

Enfin, les denrées agricoles et alimentaires de base ont ceci de particulier que la plus grande partie de leur production étant consommée dans le pays où elle a été produite ne passe aucune frontière. Les échanges internationaux de ces denrées ne portent donc que sur une petite partie de la production et de la consommation mondiale (10\% en volume pour l'ensemble de ces denrées et $15 \%$ pour les céréales par exemple). Or, ce marché international est approvisionné par les producteurs d'excédents exportables les plus compétitifs. En conséquence, en période d'excédents croissants, le prix international du blé se rapproche du coût de revient (hors transferts) des exportateurs argentins ou ukrainiens les plus compétitifs (50 à 100 dollars la tonne). Ce prix est inférieur au coût de production de la très grande majorité des agriculteurs du monde: inférieur au coût de revient des agriculteurs américains (100 à 150 dollars la tonne) qui ne pourraient pas continuer d'exporter massivement et à celui des agriculteurs européens (150 à 250 dollars), qui ne pourraient pas continuer d'approvisionner leur propre marché intérieur, s'ils ne recevaient pas, les uns et les autres, des aides publiques importantes qui leurs permettent de compenser la différence entre leurs coûts de revient et le prix international. Mais ce prix international est surtout très inférieur au coût de revient des centaines de millions de paysans, produisant moins de 1 tonne de céréales par an, qui est supérieur à 700 dollars la tonne, pour un revenu du travail de 2 dollars par jour (ce qui correspond à 1 dollar par jour par bouche à nourrir). Appauvris par la baisse des prix des productions vivrières et par celle des prix des productions d'exportation, qui suit de près celle des productions vivrières, ces centaines de millions de paysans doivent réduire ou cesser de produire et prendre le chemin du camp de réfugié, du bidonville ou de l'émigration.

\section{L'explosion momentanée des prix agricoles internationaux}

Ajoutons que ces longues périodes de baisse de prix, qui poussent à la cessation d'activités des centaines de millions de paysans pauvres, et qui découragent la production de ceux qui restent, finissent par réduire les stocks de fin de campagne, au point de provoquer une véritable explosion des prix, comme ce fut le cas en 1972. En quelques semaines, les prix du blé et des autres denrées vivrières ont triplé pour remonter aux niveaux élevés qu'ils avaient atteints au lendemain de la Seconde Guerre mondiale. Et ce qui n'a pas manqué de se produire de nouveau en 2007 du fait que les excédents à bas prix ont continué de se déverser sans protection dans les pays à faible revenu et forte dépendance vivrière.

Quand les prix sont bas, ce sont des centaines de millions de petits producteurs vendeurs de produits agricoles qui sont trop appauvris pour manger à leur faim; quand ils sont hauts, ce sont des centaines de millions de consommateurs acheteurs pauvres qui ont faim à leur tour. Dans les régions agricoles appauvries par la baisse des prix, la production couvre à peine les besoins d'autoconsommation. Les stocks sont très réduits et ils peuvent être épuisés avant la récolte suivante. Les prix peuvent alors remonter très fortement et exclure les acheteurs les plus pauvres, y compris les producteurs pauvres devenus acheteurs nets de nourriture, de tout accès à la nourriture. Sans régulation des prix, le marché ne peut subvenir aux besoins minima des uns et des autres.

La courbe du prix réel du blé sur le marché de Chicago (figure 2) illustre parfaitement ce mode de fonctionnement des marchés internationaux des denrées vivrières de base : les longues périodes de baisses des prix (1948-1972 et depuis 1979) alternent avec de courtes périodes de hauts prix $(1945-48,1972-79)$ et 2007-08).

\section{Les conséquences de la baisse des prix agricoles}

Dans les pays développés, la forte baisse des prix agricoles réels résultant de la révolution agricole contemporaine a entraîné une baisse de 
revenu des petites et moyennes exploitations qui n'avaient pas eu les moyens d'investir suffisamment pour en compenser les effets. D'année en année, des exploitations de plus en plus nombreuses se sont retrouvées dans l'incapacité de dégager un revenu familial socialement acceptable et $n^{\prime}$ ont pas été reprises, lors de la retraite de l'exploitant. Leurs meilleures terres ont alors été partagées entre les exploitations voisines en développement tandis que les moins bonnes passaient à la friche.

C'est ainsi que la très grande majorité des exploitations agricoles existant au début du $X X^{\mathrm{e}}$ siècle dans les pays développés ont cessé d'exister ; mais si, jusqu'aux années 1980, dans ces pays, les enfants d'agriculteurs quittant la terre ont généralement trouvé du travail, et des salaires croissants, dans l'industrie ou les services, il en va tout autrement pour les dizaines de millions de paysans pauvres acculés chaque année à l'exode dans les pays en développement.

Dans ces pays en effet, les paysans travaillant en culture manuelle, et confrontés à la baisse des prix agricoles, ont d'abord vu leur pouvoir $d^{\prime}$ achat baisser. La majorité d'entre eux s'est ensuite retrouvée dans l'incapacité d'investir dans un outillage performant et d'acheter des semences sélectionnées, des engrais et des pesticides. Leur développement a donc été bloqué. Puis, la baisse des prix se poursuivant, leur revenu monétaire est devenu insuffisant pour tout à la fois renouveler leur outillage et manger à leur faim. Pour mieux comprendre ce processus, considérons un céréaliculteur soudanien, andin ou himalayen disposant d'un outillage manuel et produisant, sans engrais ni pesticide, 1 tonne de grain net (semences déduites). II y a une cinquantaine d'années, un tel céréaliculteur recevait l'équivalent de 40 dollars d'aujourd'hui pour $100 \mathrm{~kg}$ de grain : il devait alors en vendre $200 \mathrm{~kg}$ pour renouveler son outillage, ses vêtements..., il lui en restait donc $800 \mathrm{~kg}$ pour nourrir, modestement, 4 personnes; en se privant un peu, il pouvait même en vendre $100 \mathrm{~kg}$ de plus pour acheter quelque outil nouveau plus efficace. II y a une vingtaine d'années, il ne recevait plus que l'équivalent de 20 dollars d'aujourd'hui pour $100 \mathrm{~kg}$ : il devait alors en vendre $400 \mathrm{~kg}$ pour renouveler son outillage et les autres biens indispensables, et il ne lui restait que $600 \mathrm{~kg}$ pour nourrir, cette fois insuffisamment, 4 personnes ; il ne pouvait donc plus acheter de nouveaux outils. Enfin, au début des années 2000, il ne recevait plus que 10 dollars pour $100 \mathrm{~kg}$ de grain : il devait donc en vendre plus de $800 \mathrm{~kg}$ pour renouveler son matériel et les autres biens indispensables, ce qui est bien sûr impossible puisqu'on ne peut nourrir 4 personnes avec $200 \mathrm{~kg}$ de grain. En fait, à ce prix-là, il est condamné à l'endettement, puis à l'exode ou à mourir sur place.

\section{Le blocage du développement}

Dans les pays pauvres cependant, la baisse des prix agricoles a d'autres conséquences: en excluant des millions de paysans et en décourageant la production de ceux qui restent, elle limite la production et accroît le déficit alimentaire ; en accélérant l'exode rural, elle amplifie le chômage et fait baisser les salaires en milieu urbain (la hiérarchie des salaires dans les différentes parties du monde suit de très près celle des revenus de la paysannerie). Partant, les revenus de la population, les recettes d'exportation et les recettes budgétaires de ces « pays agricoles pauvres » sont bien trop faibles pour qu'ils puissent se moderniser et attirer des investissements étrangers. D'où l'endettement, le surendettement, puis les politiques de réduction des dépenses, préconisées par les institutions financières internationales, qui débouchent sur la perte de légitimité et l'instabilité des gouvernements, sur l'ingouvernabilité et les conflits.

\section{Ni l'aide, ni le partage, ni les échanges internationaux ne peuvent suffire}

Ces processus additionnés contribuent à expliquer que la moitié de l'humanité se retrouve avec un pouvoir d'achat insignifiant : plus de trois milliards de personnes disposent de moins de 3 dollars par jour. Ces très bas revenus limitent la consommation alimentaire bien en dessous des besoins minima. Le supplément de revenu dont auraient besoin ces personnes pour échapper aux privations alimentaires dépasse les 2000 milliards de dollars par an. C'est vingt fois plus que l'aide publique au développement qui n'atteint même pas les 100 milliards de dollars par an. La quantité supplémentaire d'aliments, nécessaire pour supprimer la malnutrition qui frappe 2 milliards de pauvres, et la faim qui en frappe 862 millions, est égale à $30 \%$ du volume d'aliments produits et utilisés dans le monde : soit plus de cent fois le volume de l'aide alimentaire ; elle représente plus de la moitié du volume d'aliments utilisés par les 1,5 milliards de personnes les mieux (ou les plus) nourries du monde, et plus du double du volume des échanges internationaux de produits vivriers. C'est assez dire que ni l'aide alimentaire, ni l'aide publique au développement, ni le partage, ni les échanges internationaux, ne sont à la hauteur du problème. D'ailleurs, le marché n'équilibre pas la production et les besoins, il équilibre la production et la demande solvable. Et comme la demande solvable est inférieure de $30 \%$ aux besoins la production l'est aussi [6].
Plus globalement, l'insignifiance des revenus de 3 milliards de personnes, dont la majorité sont des paysans, limite beaucoup plus encore la consommation des autres biens et services, et donc les possibilités d'investissements productifs et la croissance globale tous secteurs confondus. L'économie-monde ne manque pas d'épargne, mais de débouchés.

\section{Perspectives et propositions}

En 2050, la Terre comptera vraisemblablement 9 milliards d'humains, pour plafonner autour de 9,5 milliards avant la fin du siècle. Pour nourrir tout juste correctement, sans sousalimentation ni malnutrition, une telle population, la production végétale destinée à l'alimentation des hommes et des animaux domestiques devra un peu plus que doubler dans l'ensemble du monde. Elle devra plus que tripler dans les pays en développement, plus que quintupler en Afrique, et même décupler dans certains pays de ce continent.

Pour obtenir une augmentation de production aussi importante, l'activité agricole devra être étendue et intensifiée, durablement, sur toutes les terres exploitables de la terre. Ce qui est tout à fait possible : car les terres aujourd'hui exploitées représentent environ la moitié des terres exploitables de la planète, et car les techniques connues à ce jour, dûment corrigées de leurs excès pour être durables, sont sous-utilisées dans la plus grande partie du monde. Mais, pour permettre à tous les paysans du monde d'étendre leurs cultures à toutes les terres cultivables et $d^{\prime} y$ construire des écosystèmes cultivés capables de produire, sans atteinte à l'environnement, un maximum de denrées vivrières de qualité, il faut avant tout garantir à tous ces paysans des prix assez élevés et assez stables pour qu'ils puissent vivre dignement de leur travail, investir et progresser.

Pour venir à bout de la sous-production agricole et de la sous-consommation alimentaire dans les pays à faible revenu et forte dépendance vivrière, il n'est donc pas d'autre voie que de protéger les agricultures paysannes pauvres de ces pays de la concurrence des agricultures plus compétitives. Et, dans cette perspective, il nous paraît souhaitable d'instaurer une organisation des échanges agricoles internationaux beaucoup plus équitable et beaucoup plus efficace que celle d'aujourd'hui $[3,4]$.

Une nouvelle organisation dont les principes seraient les suivants :

- établir de grands marchés communs agricoles régionaux, regroupant des pays ayant des productivités agricoles du même ordre de grandeur (Afrique de I'Ouest, Asie du Sud, Asie de l'Est, Europe de l'Ouest, Amérique du Nord...) ; 
- protéger ces marchés régionaux contre toute importation d'excédents agricoles à bas prix par des droits de douane variables, garantissant aux paysans pauvres des régions défavorisées des prix assez élevés et assez stables pour leur permettre de vivre et de se développer ;

- négocier, produit par produit, des accords internationaux fixant de manière équitable le prix d'achat et la quantité exportable consentie à chaque pays.

\section{Relever les revenus plutôt que de faire des cadeaux}

Ce relèvement des prix agricoles devra être suffisamment progressif pour limiter ses effets négatifs sur les consommateurs-acheteurs pauvres. Malgré cela, il sera sans doute nécessaire $d^{\prime}$ instaurer pendant quelque temps des politiques alimentaires. Mais, au lieu de fonder ces politiques sur la distribution de produits à bas prix, ce qui entretient la misère paysanne et réduit le marché intérieur, il conviendra de fonder ces politiques sur le soutien du pouvoir d'achat alimentaire des consommateursacheteurs pauvres, afin, au contraire, d'élargir le marché intérieur : on pourra par exemple, comme aux États-Unis, distribuer aux acheteurs nécessiteux des bons d'achat alimentaires financés par les budgets publics ou par l'aide internationale [7].

De plus, comme le relèvement des prix agricoles ne suffira pas, à lui seul, pour porter la production à la hauteur des besoins et pour promouvoir un développement agricole équilibré des différentes régions du monde, des politiques de développement agricole seront également nécessaires : accès à la terre et sécurité de la tenure (réforme agraire, statut du fermage, lois anti-cumul, aides à l'installation...) ; accès au crédit, aux intrants et aux équipements productifs; accès au marché (infrastructures de transport et de commercialisation); accès au savoir (recherche, formation, vulgarisation appropriées aux besoins et aux moyens des différentes régions et des différentes catégories de producteurs, à commencer par les plus désavantagées).

\section{RÉFÉRENCES}

1. FAO, Faostat, CDROM.

2. FAO. L'état de l'insécurité alimentaire dans le monde. FAO, Différents rapports annuels.

3. Mazoyer M, Roudart L. Histoire des agricultures du monde, du Néolithique à la crise contemporaine. Paris : Editions du Seuil, 1997, 1998; nouvelle édition Points Histoire, Seuil, 2002.

4. Griffon M. In : Révolution verte, Révolution doublement verte. Mondes en développement, tome 30. Bruxelles: Cecoeduc, 2002 : 39-44.

5. Mazoyer M, Roudart L. La fracture agricole et alimentaire mondiale. Nourrir l'humanité aujourd'hui et demain. Paris : Universalis, 2005.

6. Collomb P. Une voie étroite pour la sécurité alimentaire. Paris : FAO Economica, 1999.

7. Roudart L. In : L'alimentation dans le monde et les politiques publiques de lutte contre la faim, Mondes en développement, tome 30. Bruxelles: Cecoeduc, $2002: 9-23$. 